\section{DIE GASTROSKOPISCHE DIAGNOSE DES DIFFUSEN LYMPHOSARKOMS DES MAGENS.}

Von

Dr. Rudolf SCHINdLER.

Aus der II. madizinischen Abtejlung des Krankenhauses München-Schwabing. (Chefarzt: Prof. Dr. O. NEUBAUER.)

Das primäre Sarkom des Magens ist eine seltene Krankheit. Die klinische Diagnose wird meist auf Carcinom oder gar nicht gestellt, wenn nicht zufällig ein erbrochenes Geschwulstpartikelchen sie ermöglicht. Doch kann selbst mikroskopisch die Differentialdiagnose gegenüber dem medullären Magencarcinom schwierig sein.

Ein von uns beobachteter Fall hat uns nun gelehrt, daß diffuse Lymphosarkome des Magens im Gastroskop ein höchst charalsteristisches, von allen anderen Magenerkrankungen verschiedenes Bild bieten können, so daß sie gastroskopisch sicher diagnostizierbar sind - wenn nämlich das betreffende Bild dem Untersucher bekannt ist. Da der untenbeschriebene wohl der erste Fall von diffusem Lymphosarkom war, der zur gastroskopischen Untersuchung gelangte, so habe ich intra vitam die tichtige Diagnose nicht gestellt. Erst die Obduktion schaffte Klarheit. $\mathrm{Um}$ so wesentlicher erscheint mir die kurze Mitteilung des Falles.

Karoline W., 60 Jahre, Haushälterin.

Anamnese: Vater an Lungenleiden $\dot{\dagger}$, ein Bruder gichtleidend, sonst familiär nichts von Belang. - Alle Kinderkrankheiten, mit I 7 und I8 Jahren Nesselsucht. Vom Ende der zwanziger bis Ende der dreiBiger Jahre litt sie an Magengeschwüren, derentwegen sie mehrere Male in verschiedenen Krankenhäusern war. Damals Magenschmerzen nach dem Essen mit anschließendem Erbrechen und Magenblutungen. - - Später einige Male Grippe.

Anfang Juli r92 I bekam sie - angeblich ohne Diätfehler wiederholt Erbrechen und Diarrhöen, die einen Tag anhielten. Trotz Bettruhe und Diät blieb Appetitlosigkeit und Mattigkeit noch längere Zeit bestehen. Bald stellten sich Schmerzen in der Magengegend ein, verbunden mit saurem Aufstoßen. Sie waren unabhängig von den Mahlzeiten, nachts häufiger als am Tage, dauerten etwa Io Minuten. Durch Flüssigkeitsaufnahme verschlimmerten sie sich in der Regel. Dabei hatte die Kranke das Gefühl eines Hindernisses in der Speiseröhre. Feste Nahrung machte weniger Beschwerden. Erbrochen wurde nie. Die Kranke nahm $a b$ und wurde immer matter. Stuhl jetzt regelmäßig, meist breiig, Appetit gering.

Befund (6. X. 1921): Abgemagerte, blasse Kranke. Kein Fieber. - Zunge feucht, foetor ex ore. Zähne in sehr schlechtem Zustand. Unterleib weich, schlaffe Bauchdecken; um den Nabel Druclsschmerz. - Sonst Organe o. B. Erste Stuhluntersuchung ergibt negative Blutreaktionen. - Magensaft (nach Probefrühstück): Sanguis ++ , Freie $\mathrm{HCl} \varnothing$, Defizit 36 , Gebundene Säure 5, Lab $\varnothing$, Pepsin + (?), Milchsäure $\varnothing$, mikroskopisch alle Arten von Bakterien, massenhafte Sarcine, Leukocyten ++ , Erythrocyten + .

Röntgenologisch: Magenkörper schlaff, Fundus gesenkt, im Duodenum deutliche starke Stauung, Antrumperistaltik mäßig stark.

I4. X. I921. 1. Gastroskopie: Der Pylorus ist ein auffällig enger Schlitz, arbeitet langsam, aber normal, schließt sich völlig. Die Schleimhaut der kleinen Kurvatur zeigt ausgedehnt flächenförmige submuköse Hämorrhagien. An einet Stelle durchbricht das Blut die Mucosa, ohne da.ß hier eine eigentliche Geschwürsbildung zu sehen wäre; es hängt stalaktitenförmig herab, von der Kuppe des Vorsprungs fällt Tropfen nach Tropfen flüssigen Bluts in die freie Magenhöhle hinab. - Wo die Schleimhaut nicht blutdurchtränkt ist, sieht sie etwas aufgelockert aus und ist mit etwas weiBlichem, zähem Schleim bedeckt. Wegen der Blutung wird die Gastroskopie unterbrochen. - Gastroskopische Diagnose: Gastritis chronica hypertrophicans haemorrhagica (?)

Nach der Gastroskopie wird chemisch Blut im Stuhl nachgewiesen. Bei häufigen Nachuntersuchungen wird es bald vermißt, bald gefunden. Blutbild. Hgl: $80 \%$. Erythroc. 3,8 Mill. Färbeindex 1.07. Leukocyten: 7900, davon Neutrophile polynucl. $58 \%$, eosinophile $5 \%$, Mononucl. usw. $4 \%$, Lymphocyten $33 \%$.

Das subjektive Befinden der Kranken besserte sich bedeutend, das Gewicht hob sich bei Schonungsdiät und Darreichung von Salzsäurepepsin.

Dennoch war sie nach 4 Monaten mit einer zweiten gastroskopischen Untersuchung einverstanden. Das war mir darum besonders lieb, weil ich fürchtete, die Blutung bei der ersten Untersuchung sei eine artefizielle, durch das Instrument in entzündlich veränderter Schleimhaut gesetzte gewesen. Die erneute Untersuchung zeigte die Grundlosigkeit dieser Annahme.

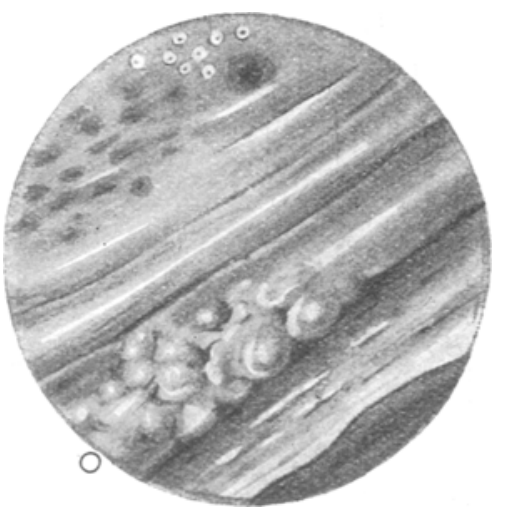

Erklïrung: Gastroskopisches Bild eines diffusen Lymphosarkoms des Magens. Patient in linker Seitenlage, das Prisma der Optik ist nach der Korpuhöhle zu gewenđet. In ihr groBer Flüssigkeitssee. Darüber starre, mit Höckern und Knollen besetzte Leisten. An der Vorderwand grobe Fleckung der Schleimhaut, lufthaltiga Schleimblasen, Blutungen. Rechts unten eine Falte der Hinterwand.

2. Gastroskopie: Magen enthält enorme Mengen von blutigem Schleim, so daß das ganze Gastroskoprohr voll Flüssigkeit ist. Dennoch gelingt es, ein klares Bild aller Magenteile zu bekommen. Die Schleimhaut zeigt überall, mit Ausnahme des Antrums und des Pylorus, der träge arbeitet, die schwersten Veränderungen. Sie ist weich, aufgelockert, an vielen Stellen mit massenhaft schaumigem Schleim belegt. Ưberall grobe Fleckung. Am meisten in die Augen fällt, daß die Schleimhaut - diesmal nicht nur an der kleinen Kurvatur, sondern allerorten - mit riesigen, flächenförmigen Hämorrhagien durchtränkt ist, die sie teilweise warzig oder halbkugelig hervortreiben. - An der Vorderwand der großen Kurvatur der Korpushöhle Bildung starrer Leisten und Bänder, die höckrig-knollige Beschaffenheit zeigen. Wären die übrigen diffusen Schleimhautveränderangen nicht. da, so würde man unbedingt an Carcinom denken. - An wenigen ganz circumscripten Stellen der Korpushöhle zeigt die Schleimhaut keine Veränderungen.

Wäre nicht die ungeheure Ausdehnung der Veränderungen, so hätte ein Carcinom diagnnstiziert werden müssen; so mußte ich mich zu der Diagnose einer Gastritis chronica proliferans haemorrhagica ungewöhnlicher Art entschließen.

Dennoch wurde der Kranken Probelaparotomie angeraten, die sie aber ablehnte. Sie verließ bei subjektivem Wohlbefinden das Krankenhaus. Objektiv war der Magensaft- und Stuhlbefund unverändert geblieben.

Nach 4 Monaten (am 23. V. I922) Wiederaufnahme in desolatem Zustand. Drohende Peritonitis; hohes Fieber, kugelig vorgewölbter Leib. Stuhl: Sanguis +. Blutbild: Hgl. $51 \%$, Erythrocyten 2,7 Millionen, Färbeindex 0,85. Leukocyten: 7240 , davon Neutr. polyn. $85,5 \%$, Mastzellen $0,5 \%$, Mononucl. $3 \%$, Lymphocyten II $\%$. Röntgenologisch diesmal ,,Verdacht auf extraventriculären Tumor, der auf die kleine Kurvatur bzw. das Duodenum Druckerscheinungen ausübt." Befund (Prof. Dr. Grashey): Magen quer bogenförmig; an der kleinen Kurvatur anscheinend durch einen Tumor eingebuchtet. Duodenum deutlich stark gestaut. Pylorus etwas nach rechts vetzogen. - Nach $3^{1 / 2}$ Stunden Magen leer. - Gastroskopie wegen des Fiebers und der peritonealen Reizerscheinungen kontraindiziert. - II. VI. I922. Exitus.

Obdultion (starke Fäulnis): Der herauspräparierte Magen hat Dreimannsfaustgröße und walzenförmige Gestalt, welch letztere durch die von außen fühlbare, beträchtliche Verstärkung fast der gesamten Magenwand bedingt.ist. Nach dem Aufschneiden zeigt sich die Magenschleimhaut zu $9 / 10$ aus im ganzen morschen, an ihrer Oberfläche bräunlich-nekrotisierenden, nach dem Abheben der $1-2 \mathrm{~mm}$ dicken, nekrotischen Schicht markig-weichen, weißen bis gèlblichen, bis intensiv rot tingierten Massen bestehend. Der Pylorus selbst, sowie vereinzelte dreimarkstückgroße Schleimhautzonen zeigen nicht nekrotische, sondern nu* gering verdickte Schleimhaut usw.

Anatomische Diagnose (nach mikroskopischer Untersuchung): Ausgedehntes. oberflächlich nekrotisierendes Lymphosarkom des Magens, mit Metastasen in den perigastrischen, peripankreatischen und periaortischen Lymphdrüsen. Ausgedehnte Perforation des Tumors an der Vorderwand des Magens mit partieller Abdeckung der Perforation durch $Z$ werchfell und Netz. Sekundäre allgemeine Anämie. Thrombose beider Schenkelvenen. Odeme der Beine und Labien. Nebenbefunde. (Prof. Dr. S. Oberndorfire.) 
Außer seiner Seltenheit bietet der Fall klinisch wie anatomisch nicht viel Bemerkenswertes. $\mathrm{Zu}$ beachten ist die anamnestische Angabe von Magengeschwüren, $3^{\circ}$ Jahre vor Beginn der jetzigen Krankheit. Daß subjektive Besserung bei objektiv enormer Verschlimmerung angetreten war, kann uns kaum erstaunen. Wir wissen ja, daß die Magenfunktion rasch ersetzt werden kann. Das Blutbild zeigte die für manche Lymphosarkome charakteristische Lymphopenie erst kurz vor dem Ende. Mit den üblichen klinischen Methoden war die Diagnose nicht zu stellen. Auch die Röntgenuntersuchung muBte zu einer Zeit versagen, wo die Gastroskopie schon einen sehr ausgesprochenen Befund lieferte, da der Antrumschlauch gut arbeitete, die Infiltration der Magenwandung noch gering war.

Von ausschlaggebender Bedeutung für die Diagnose des diffusen Lymphosarkoms ist die Untersuchung mit dem Gastroskop ${ }^{1}$ ). Sie lieferte schon in frühen Stadien der Krankheit Bilder, wie sie bei keiner anderen Magenkrankheit vorkommen ${ }^{2}$ ). Es bleibt freilich abzuwarten, ob alle Fälle vom Lymphosarkom das gleiche gastroskopische Bild geben werden. - Zunächst bietet die Schleimhaut zwar das Aussehen der Gastritis hypertrophicans, sie ist samtartig, locker, geschwollen. Aber wenn auch bei jener Krankheit Hämorrhagien häufig sind, so erreichen sie doch niemals die ungeheure beetartige Ausdehnung wie beim Lymphosarkom. Ebenso habe ich bei keiner anderen Magenkrankheit ein Durchbrechen submuköser Hämorrhagien in die freie Magenhöhle beobachtet. In späteren Stadien nehmen die Hämorrhagien noch sehr an Umfang zu, es tritt die Bildung charakteristischer starrer Leisten und Bänder hinzu, die höckrig-knollige Beschaffenheit zeigen. Im Gegensatz dazu sind die Vorwölbungen der Gastritis proliferans s. polyposa, einer ebenfalls seltenen Krankheit, an die ich fälschlicherweise dachte, von lockerem, bläulich durchscheinendem Aussehen.

Bei Kenntnis des Bildes ist also die Diagnose des diffusen Lymphosarkoms des Magens durch die Untersuchung mit dem Magenspiegel möglich, was für Prognose und Therapie von allergrößter Bedeutung sein kann.

\section{ÜBER DAS DIAGNOSTISCHE TUBERKULIN NACH MORO.}

Von

\section{Dr. KRETSCHMER.}

Aus der III. med, Universitätsklinik Berlin. (Direktor: Geheimrat Prof. Dr. GOLDSCHEIDER.)

In einer kürzlich erschienenen Arbeit über die bisherigen Erfahrungen mit dem ,diagnostischen Tuberkulin" (DT.) erklärt SCHARNkE nach Anführung mehrerer Autoren, die günstige Resultate mit dem MoRoschen Tuberkulin erzielt hatten, es nicht beurteilen zu können, wie ich zu meinem auffallenden Resultat gelangt wäre, indem ich in einer kurzen Mitteilung feststellte, daß eine Uberlegenheit des diagnostischen Tuberkulins gegenüber dem Alttuberkulin (AT.) Höchst nicht bestände. Meine damaligen Ergebnisse der vergleichenden Impfungen waren unter I 69 positiven Reaktionen von 300 geimpften Fällen, I5 $(8,8 \%)$ nur positiv mit AT., $9(5,3 \%)$ nur positiv mit DT., dagegen I 8 (I0,6\%) stärker auf AT., 24 $\left(\mathrm{I}_{4}, \mathrm{I} \%\right.$ ) stärker auf DT. reagierend. Die Prozentzahlen der mit einem der beiden Tuberkuline stärker reagierenden Fälle war alsofür AT. und DT. gleich, wenn auch mitAT. ein größerer Prozentsatz eine Reaktion gab, wo DT. versagte.

Eine weitere Arbeit von LIPPMANN, der ebenfalls in noch stärkerem Maße als ich bessere Resultate mit dem AT. Höchst gegenüber dem DT. Moro erzielte, fertigt SCHARnkE mit der Erklärung ab, daß seine abweichenden Ergebnisse sich durch die verschiedene Impfmethode erklärten, indem LIPPMANN statt des Pirquetbohrers sich zu seinen Impfungen

1) Sie wurde mit dem von mir angegebenen Modell ausgeführt (Fabrikant: Reiniger, Gebbert und Schall, München, Mathildenstr. 13; vgl. Münch. med. Wochenschr. 1922, Nr. 535).

2) Dreifarbige Abbildungen der gastrosk. Bilder des hier beschriebenen Falles sind in meinem im Erscheinen begriffenen A tlas und Lehrbuch der Gastroskopie enthalten. einer spitzen Impflanzette bediente. Ich glaube nicht, daß sich dadurch die für das diagnostische Tuberkulin ungünstigen Resultate erklären lassen, denn das wesentliche der cutanen Impfung ist doch eine Verletzung der Epidermis und eine solche wird doch schließlich mit einer Impflanzette ebenso gut erreicht wie mit dem Pirquetbohrer oder wie es auch neuerdings empfohlen wird, sogar mit einem Stückchen Sandpapier.

Auch RöckEMANN kann keine wesentlichen Vorteile des diagnostischen Tuberkulins im Vergleich mit dem AT. Höchst feststellen. Von der verhältnismäßig kleinen Zahl seiner positiv reagierenden Fälle war die Impfung mit AT. stärker I 7 mal, mit DT. I6 mal, mit beiden gleich I4 mal; er stellt ferner fest, daB die Reaktion mit AT. meist nach 24 Stunden, die mit DT. nach $4^{8}$ Stunden stärker war und daß die Reaktion des DT. langsamer abklang als die von AT. Ferner fand RöckEMANN bei Impfungen mit verdünntem DT.. $\mathrm{da} B$ die Reaktionen gelegentlich stärker ausfielen als mit dem konzentrierten DT., so daß man also den stärkeren Konzentrationsgrad des diagnostischen Tuberkulins nicht als einen Vorzug ansehen könne, da die Resorptionsverhältnisse ganz andere sein müßten als die des AT.

An unserer Klinik hat ScHREIBER nach Abschluß meiner Arbeit die vergleichenden Impfungen mit AT. und DT. fortgesetzt, wobei die Resultate von allen auf der Kinderabteilung arbeitenden Kollegen mit geprüft wurden. Seine Ergebnisse, die er in seiner zur Zeit im Druck befindlichen Dissertation niedergelegt hat, waren bei i 76 Impfungen: positiv und gleich mit AT. und DT. 26 Fälle, nur mit AT. positiv I 4 Fälle, nur mit DT. positiv 8 Fälle, mit AT. stärker reagierend 2I Fälle, mit DT. stärker I 5 Fälle. Wir kamen also auch bei diesei Untersuchungsreihe zu dem Ergebnis, daß das diagnostische Tuberkulin keine wesentlichen Vorzüge gegenüber dem AT. Höchst hat, wenn auch mit jedem der beiden Tuberkuline manche Fälie allein eine Reaktion geben. Dies ist auch der Grund, weshalb wir seit den ersten vergleichenden Impfungen in allen Fällen beide Tuberkuline anwenden, um mit Sicherheit aile tuberkulösen Kinder festzustellen. Eine Anderung in den Prozentzahlen der nur auf ein Tuberkulin oder auf eines der beiden Tuberkuline stärker reagierenden Kinder hat sich. jedoch nicht ergeben.

Bei der Untersuchung der Gründe, die unsere von den Resultaten anderer Kliniken abweichenden Ergebnisse verursachen könnten, haben wir gefunden, đaß möglicherweise der Grund in der Anordnung der Impfstellen der beiden Tuberkuline liegen könnte. Bekanntlich ist bei vergleichenden Impfungen an verschiedenen Stellen des Armes mit demselben Tuberkulin im allgemeinen die Reaktion an der proximal gelegenen Impfstelle stärker als aǹ der distalen. Wir haben daher eine Zeitlang bei allen geimpften Kindern an beiden Unterarmen je zwei Impfungen und je eine Kontrolle angelegt, und zwar am rechten Arm die obere mit AT. die untere mit DT. beschickt, am linken Arm umgekehrt. Dabei zeigte sich, wie wir erwarteten, $\mathrm{da} B$ fast immer bei Differenzen die oberen Impfstellen stärker reagierten als die unteren bzw. daß auf der rechten Seite nur die obere Impfstelle mit AT. auf dem linken nur die mit DT. eine positive Reaktion ergab. Kontrolliert wurden sämtliche Fälle nach 48 Stunden, da wir die Beobachtung gemacht haben, daß dann die Reaktion fast immer voll ausgebildet ist, während bei vielen Impfungen nach 24 Stunden noch keine Reaktion zu sehen ist. $\mathrm{Zu}$ mehrmaliger Kontrolle die Kinder zu bestellen, verbietet sich hier durch die großen Entfernungen und hohen Fahrpreise.

Nach unseren Ergebnissen erscheint es daher notwendig zum Vergleich verschiedener Resultate mit Tuberkulinimpfungen immer die Angabe zu machen, an welchen Stellen des Armes sich die Impfstellen der zu vergleichenden Tuberkuline befanden, zweckmäßig wird man die Impfungen stets an beiden Armen in umgekehrter Reihenfolge für die zu vergleichenden Tuberkuline vornehmen.

Liter a tur: ScharnKe, Klin. Wochenschr: Nr. 27. I922 (Literaturübersicht). - RöckEMANN, Monatsschr. f. Kinderheilk, 23, H. 2. - Schreiber, Inaug. Diss. Berlin I922. 